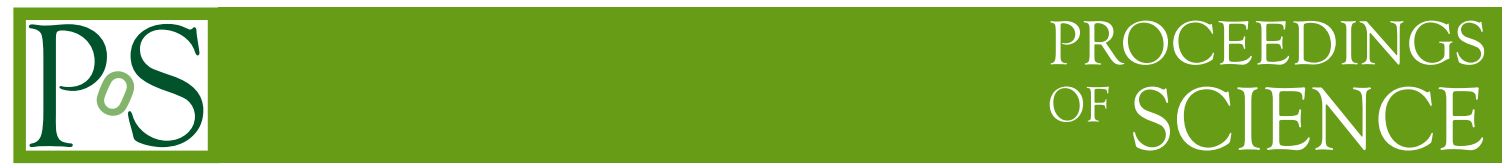

\title{
Beauty-hadron spectroscopy at LHCb
}

\section{Hongjie $\mathbf{M u}^{*}$ on behalf of the LHCb collaboration}

Center for High Energy Physics, Tsinghua University, Beijing, China

E-mail: hongjie.mu@cern.ch

The latest results of conventional beauty-hadron spectroscopy from the LHCb experiment are introduced in this proceeding. These results contain the observation of new excited $B_{s}^{0}$ states in $B^{+} K^{-}$final state, the observation of a new excited $\Xi_{b}^{0}$ state in $\Xi_{b}^{-} \pi^{+}$final state, the observation of two new exited $\Xi_{b}^{0}$ states decaying to $\Lambda_{b}^{0} K^{-} \pi^{+}$and a search for doubly heavy baryons $\Xi_{b c}^{0}$ and $\Omega_{b c}^{0}$ decaying to $\Lambda_{c}^{+} \pi^{-}$and $\Xi_{c}^{+} \pi^{-}$.

*** The European Physical Society Conference on High Energy Physics (EPS-HEP2021), ***

*** 26-30 July 2021 ***

*** Online conference, jointly organized by Universität Hamburg and the research center DESY ***

${ }^{*}$ Speaker 


\section{Introduction}

The constituent quark model $[1,2]$ was proposed for classifying and describing the hadrons composed by light quarks $(u, d, s)$ and later extended to the heavy flavor hadrons containing $b$ or $c$ quarks. The spectroscopy of hadrons containing a $b$ quark (beauty-hadron) provides important information understanding the hadronic structure and nonperturbative quantum chromodynamics (QCD). This proceeding focuses on the recent results of beauty-hadron spectroscopy from the $\mathrm{LHCb}$ experiment.

\section{Observation of new excited $B_{s}^{0}$ states}

Potential models exploiting heavy-quark symmetry [3] are used to calculate properties of $B_{s}^{0}$ meson $(b \bar{s})$. It is still difficult to precisely predict masses and widths of $B_{s}^{0}$ mesons. Therefore, the experimental results could provide more inputs and constraints for theories. A peaking structure is observed in the $B^{+} K^{-}$mass spectrum [4] with the full $9 \mathrm{fb}^{-1}$ LHCb dataset, which is interpreted as the overlapping excited $B_{S}^{0}$ states. The inclusion of charge-conjugated processes is implied and natural units with $\hbar=c=1$ are used throughout this proceeding.

The $B^{+}$candidates are reconstructed with the decays $B^{+} \rightarrow J / \psi K^{+}$and $B^{+} \rightarrow \bar{D}^{0} \pi^{+}$, where the $J / \psi$ meson subsequently decays to the $\mu^{+} \mu^{-}$final state and the $\bar{D}^{0}$ meson decays to the $K^{+} \pi^{-}$ final state. The selected $B^{+}$candidates are further combined with a $K^{-}$candidate from the primary $p p$ interaction vertex (prompt kaon) to form the $B_{s}^{* * 0}$ candidates. The $B_{s}^{* * 0}$ candidates are studied in bins of transverse momentum $\left(p_{\mathrm{T}}\right)$ of the prompt kaon, as it is a strongest discriminant between resonant signal and the combinatorial background. The spectrum of mass difference, $\Delta m \equiv m_{B^{+} K^{-}}-m_{B^{+}}-m_{K^{-}}$, is shown in Fig. 1 . A clear excess at approximately $300 \mathrm{MeV}$ above the mass threshold can be seen in the $\Delta m$ spectrum, especially in the high $p_{\mathrm{T}}$ region. Two models of signal decay are considered: one assuming the $B_{s}^{* * 0}$ state directly decays to the $B^{+} K^{-}$final state and for the other one, it decays through intermediate $B^{*+}$ meson, which further decays to the $B^{+} \gamma$ final state. The latter case result in approximately $45 \mathrm{MeV}$ shift of peak position due to the unreconstructed photon. The local significance is lager than 20 standard deviations $(\sigma)$ for one-peak fit with respect to the no-peak hypothesis and $7.7 \sigma$ for the two-peak fit with respect to the one-peak hypothesis. With the two-peak hypothesis, the masses and widths of two states decaying directly to the $B^{+} K^{-}$system are determined to be $m_{1}=6063.5 \pm 1.2$ (stat) \pm 0.8 (syst) MeV, $\Gamma_{1}=26 \pm 4$ (stat) \pm 4 (syst) MeV, $m_{2}=6114 \pm 3$ (stat) \pm 5 (syst) MeV, $\Gamma_{2}=66 \pm 18$ (stat) \pm 21 (syst) MeV. If the decay proceeds through $B^{*+} K^{-}$, the corresponding masses and widths are also measured [4]. A single resonance that decays in both the $B^{+} K^{-}$and $B^{*+} K^{-}$channels is disfavored by more than $2 \sigma$ with respect to the two-state hypothesis and cannot be completely excluded. The production ratio relative to the $B_{s 2}^{* 0}$ meson is determined to be $R=0.87 \pm 0.15$ (stat) \pm 0.19 (syst), where the production ratio is defined as the product of the cross-section times branching fractions of the new states divided by the corresponding product for $B_{s 2}^{* 0}$.

\section{Observation of a new $\Xi_{b}^{0}$ state}

Recently, the LHCb collaboration reported the observation of $\Xi_{b}(6227)^{-}$baryon in the $\Lambda_{b}^{0} K^{-}$ and $\Xi_{b}^{0} \pi^{-}$mass spectrum [5]. The isospin partner of the $\Xi_{b}(6227)^{-}$state is expected to decay 

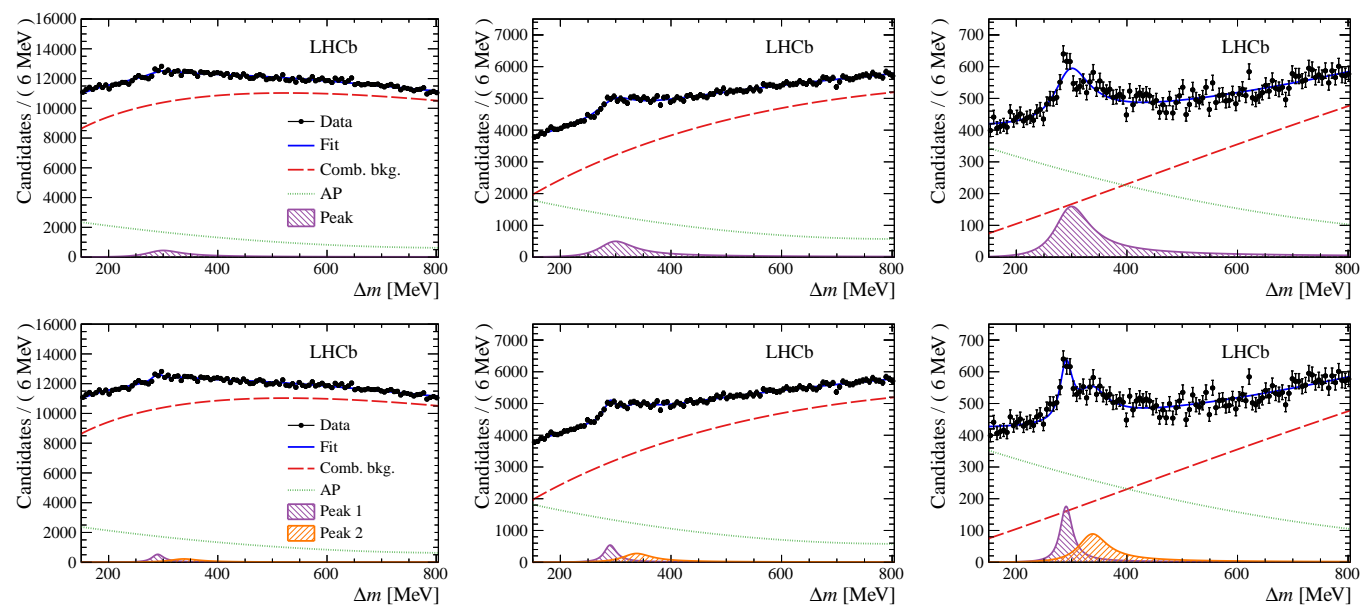

Figure 1: The $B^{+} K^{-}$mass difference distributions in data, overlaid with the fit: (top) one-peak hypothesis and (bottom) two-peak hypothesis. In each row, the columns are the candidates with prompt kaon $p_{\mathrm{T}}$ : (left) $0.5<p_{\mathrm{T}}<1 \mathrm{GeV}$, (middle) $1<p_{\mathrm{T}}<2 \mathrm{GeV}$ and (right) $p_{\mathrm{T}}>2 \mathrm{GeV}$.

through $\Xi_{b}^{-} \pi^{+}$mode. In this analysis, the observation of a new excited $\Xi_{b}^{0}$ resonance decaying to the $\Xi_{b}^{-} \pi^{+}$final state with the full LHCb dataset is presented [6]. With the enlarged data sample, the measurement of $\Xi_{b}^{-}$and $\Xi_{b}(6227)^{-}$states is also updated.

The $\Xi_{b}^{-}$candidates are reconstructed with the $\Xi_{c}^{0} \pi^{-}$and $\Xi_{c}^{0} \pi^{-} \pi^{+} \pi^{-}$decays, while the $\Lambda_{b}^{0}$ candidates reconstructed with $\Lambda_{c}^{+} \pi^{-}$and $\Lambda_{c}^{+} \pi^{-} \pi^{+} \pi^{-}$final states. The charm baryons, $\Xi_{c}^{0}$ and $\Lambda_{c}^{+}$, are reconstructed with the decays $\Xi_{c}^{0} \rightarrow p K^{-} K^{-} \pi^{+}$and $\Lambda_{c}^{+} \rightarrow p K^{-} \pi^{+}$. The $\Xi_{b}(6227)^{0}$ candidates are formed by combining a $\Xi_{b}^{-}$candidate with a prompt $\pi^{+}$candidate, while $\Xi_{b}(6227)^{-}$ candidates formed by combining $\Lambda_{b}^{0}$ and prompt $K^{-}$candidates. The spectrum of mass difference, $\delta M \equiv M\left(\Xi_{b}^{-} \pi^{+}\right)-M\left(\Xi_{b}^{-}\right)$, of right-sign $\left(\Xi_{b}^{-} \pi^{+}\right)$and wrong-sign $\left(\Xi_{b}^{-} \pi^{-}\right)$combinations is shown in Fig. 2. A clear signal is observed in the right-sign final state, while there are no significant structure in the wrong-sign mass spectrum. The statistical significance of the signal is about $10 \sigma$. The mass and width of $\Xi_{b}(6227)^{0}$ state are $m\left(\Xi_{b}(6227)^{0}\right)=6227.1_{-1.5}^{+1.4} \pm 0.5 \mathrm{MeV}$ and $\Gamma\left(\Xi_{b}(6227)^{0}\right)=18.6_{-4.1}^{+5.0} \pm 1.4 \mathrm{MeV}$. The relative production rate of the $\Xi_{b}(6227)^{0}$ state at $\sqrt{s}=13 \mathrm{TeV}$ is measured to be $R\left(\Xi_{b}^{-} \pi^{+}\right)=0.045 \pm 0.008 \pm 0.004$, where $R\left(\Xi_{b}^{-} \pi^{+}\right)$is defined as the ratio of signal yield of $\Xi_{b}(6227)^{0}$ and $\Xi_{b}^{-}$baryon divided by the relative efficiency between the $\Xi_{b}(6227)^{0}$ and $\Xi_{b}^{-}$selections. The mass and with of $\Xi_{b}(6227)^{-}$state, along with the mass of the $\Xi_{b}^{-}$baryon, are also measured with better precision.

\section{Observation of two new excited $\Xi_{b}^{0}$ states decaying to $\Lambda_{b}^{0} K^{-} \pi^{+}$}

The LHCb collaboration reported the observation of two excited $\Lambda_{b}^{0}$ resonances in the $\Lambda_{b}^{0} \pi^{+} \pi^{-}$ system [7], which are consistent with the 1D $\Lambda_{b}^{0}$ states [8]. This observation motivates the investigation of $1 \mathrm{D} \Xi_{b}^{0}$ states, as the $\Lambda_{b}^{0}$ and $\Xi_{b}^{0}$ baryons have similar properties due to the approximate $S U$ (3) flavor symmetry [8]. The two $1 \mathrm{D} \Xi_{b}^{0}$ states are predicted to decay through $\Sigma_{b}^{(*)} K$ modes [8], which result in a $\Lambda_{b}^{0} K^{-} \pi^{+}$final state. Two new excited $\Xi_{b}^{0}$ states are observed in the $\Lambda_{b}^{0} K^{-} \pi^{+}$system with the LHCb dataset at $\sqrt{s}=13 \mathrm{TeV}$ [9]. 

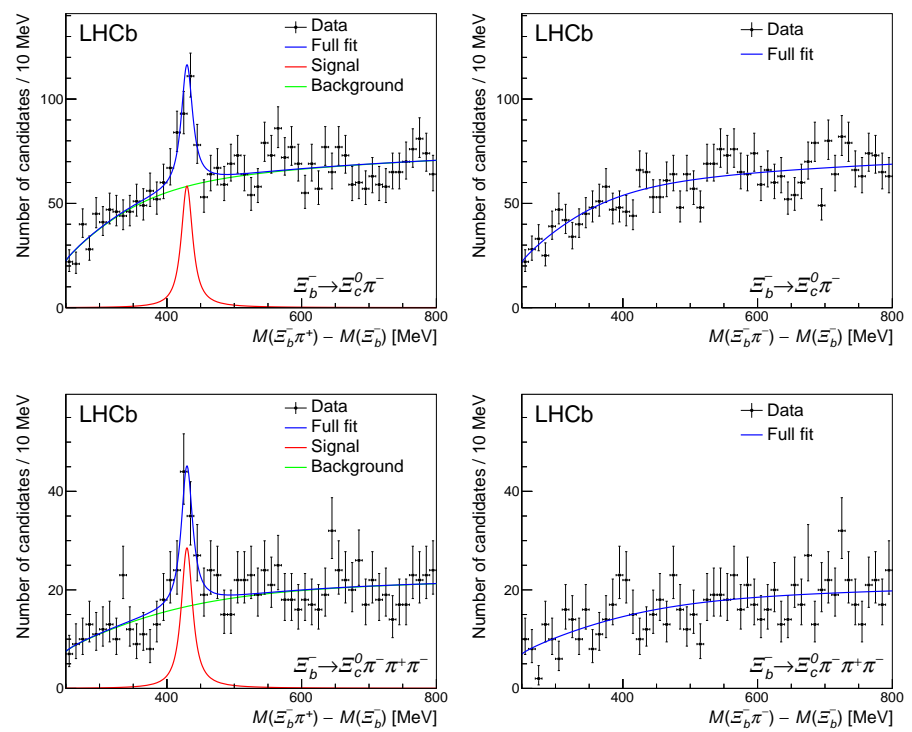

Figure 2: Distributions of mass difference $\delta M$ of (left) right-sign and (right) wrong-sign $\Xi_{b}(6227)^{0}$ candidates, with (top) $\Xi_{b}^{-} \rightarrow \Xi_{c}^{0} \pi^{-}$and (bottom) $\Xi_{b}^{-} \rightarrow \Xi_{c}^{0} \pi^{-} \pi^{+} \pi^{-}$decays.

The $\Lambda_{b}^{0}$ candidates are reconstructed with the $\Lambda_{c}^{+} \pi^{-}$and $\Lambda_{c}^{+} \pi^{-} \pi^{+} \pi^{-}$decays, where the $\Lambda_{c}^{+}$ baryon subsequently decays to the $p K^{-} \pi^{+}$final state. The selected $\Lambda_{b}^{0}$ candidates are combined with a prompt kaon and pion to form the $\Lambda_{b}^{0} K \pi$ candidates. To improve the mass resolution, the mass of $\Lambda_{b}^{0} K^{-} \pi^{+}$candidates is redefined with the reconstructed mass difference between $\Lambda_{b}^{0} K^{-} \pi^{+}$ and $\Lambda_{b}^{0}$ candidates plus the measured $\Lambda_{b}^{0}$ mass. The mass spectrum of right-sign $\left(\Lambda_{b}^{0} K^{-} \pi^{+}\right)$and wrong-sign $\left(\Lambda_{b}^{0} K^{+} \pi^{-}\right)$combinations is shown in Fig.3. Two narrow peaks can be seen in the $\Lambda_{b}^{0} K^{-} \pi^{+}$mass spectrum, while no significant peaking structure is visible in the $\Lambda_{b}^{0} K^{+} \pi^{-}$system. The significance of two-peak hypothesis is larger than $9 \sigma(5 \sigma)$ compared to the no-peak (one-peak) hypothesis. The masses of these two states are $m\left(\Xi_{b}(6327)^{0}\right)=6327.28_{-0.21}^{+0.23} \pm 0.08 \pm 0.24 \mathrm{MeV}$, $m\left(\Xi_{b}(6333)^{0}\right)=6332.69_{-0.18}^{+0.17} \pm 0.03 \pm 0.22 \mathrm{MeV}$, where the uncertainties are statistical, systematic and due to $\Lambda_{b}^{0}$ mass measurement. The corresponding widths are consistent with zero, and upper limits at $90 \%(95 \%)$ credibility level are set, $\Gamma\left(\Xi_{b}(6327)^{0}\right)<2.20(2.56) \mathrm{MeV}$, $\Gamma\left(\Xi_{b}(6333)^{0}\right)<1.55(1.85) \mathrm{MeV}$. The resonant structure in the excited $\Xi_{b}^{0}$ decays is shown in Fig. 4. The $\Xi_{b}(6327)^{0}$ state predominantly decays to $\Sigma_{b}^{+} K^{-}$. About half of the $\Xi_{b}(6333)^{0}$ baryons decay without $\Lambda_{b}^{0} \pi^{+}$resonances, while the rest is dominated the decay through $\Sigma_{b}^{*+}$ resonance. The masses, widths and decay patterns of the two observed $\Xi_{b}^{0}$ states are consistent with the predictions [8] for a doublet of $1 \mathrm{D} \Xi_{b}^{0}$ states.

\section{Search for doubly heavy baryons $\Xi_{b c}^{0}$ and $\Omega_{b c}^{0}$ decaying to $\Lambda_{c}^{+} \pi^{-}$and $\Xi_{c}^{+} \pi^{-}$}

No baryons containing $b$ and $c$ quarks have been observed experimentally. An observation would improve the understanding of the quark structure inside baryons. This analysis presents a search for $\Xi_{b c}^{0}$ and $\Omega_{b c}^{0}$ decaying to $\Lambda_{c}^{+} \pi^{-}$and $\Xi_{c}^{+} \pi^{-}$with the LHCb dataset collected in 2016$2018[10]$. 

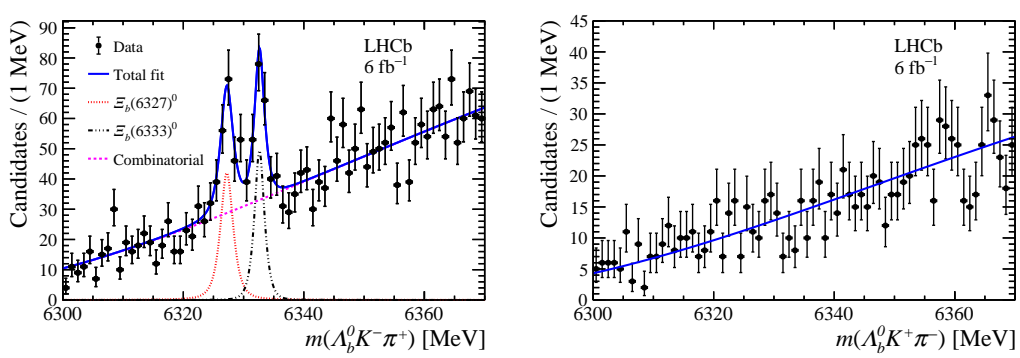

Figure 3: Invariant mass distributions of $\Lambda_{b}^{0} K \pi$ candidates of (left) right-sign and (right) wrong-sign samples.
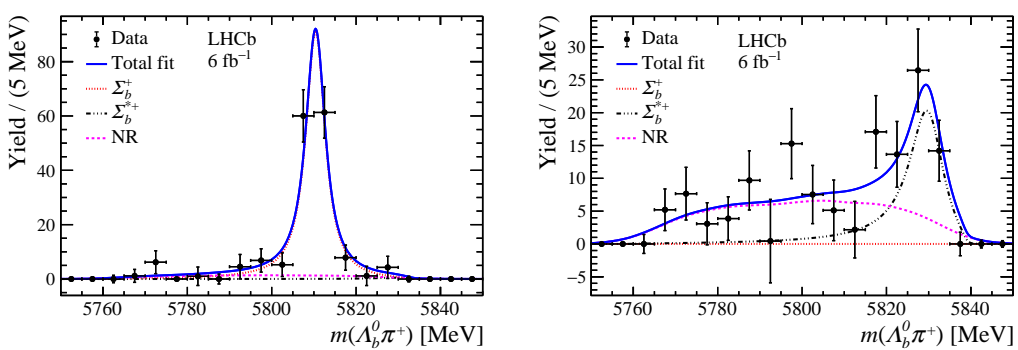

Figure 4: Signal yields of the (left) $\Xi_{b}(6327)^{0}$ and (right) $\Xi_{b}(6333)^{0}$ states in bins of $\Lambda_{b}^{0} \pi$ mass spectrum.
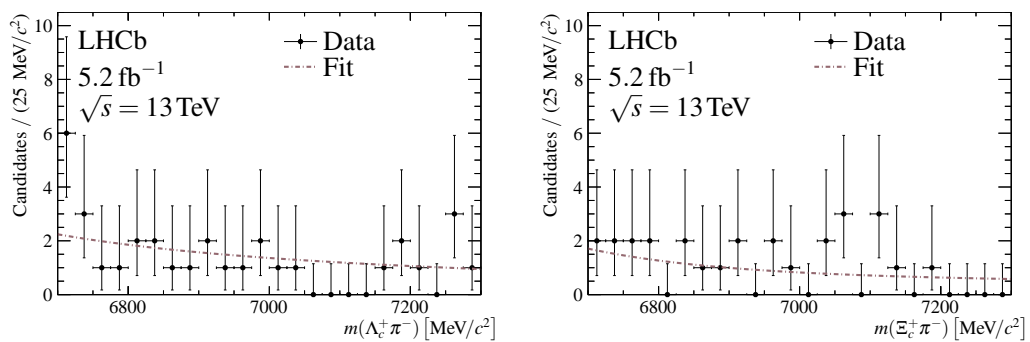

Figure 5: Invariant mass distributions of selected (left) $H_{b c}^{0} \rightarrow \Lambda_{c}^{+} \pi^{-}$and (right) $H_{b c}^{0} \rightarrow \Xi_{c}^{+} \pi^{-}$candidates.

The $\Xi_{b c}^{0}$ and $\Omega_{b c}^{0}$ baryons have similar properties and are denoted as $H_{b c}^{0}$ hereafter. The $\Lambda_{c}^{+}$ and $\Xi_{c}^{+}$candidates are reconstructed with the $p K^{-} \pi^{+}$final state. A pion is combined with the $\Lambda_{c}^{+}\left(\Xi_{c}^{+}\right)$candidate to form $H_{b c}^{0}$ candidate. The invariant mass distribution of $H_{b c}^{0}$ candidates are shown in Fig. 5. No significant excess is observed across the searched mass range. The decays of $\Lambda_{b}^{0} \rightarrow \Lambda_{c}^{+} \pi^{-}$and $\Xi_{b}^{0} \rightarrow \Xi_{c}^{+} \pi^{-}$are selected as the control channel to measure the relative production ratio of $H_{b c}^{0} \rightarrow \Lambda_{c}^{+} \pi^{-}$and $H_{b c}^{0} \rightarrow \Xi_{c}^{+} \pi^{-}$decays. The production ratio $\mathcal{R}$ is defined as the $H_{b c}^{0}$ production cross-section multiplied by the branching fraction of $H_{b c}^{0} \rightarrow \Lambda_{c}^{+} \pi^{-}$and $H_{b c}^{0} \rightarrow \Xi_{c}^{+} \pi^{-}$ decays relative to that of the $\Lambda_{b}^{0}\left(\Xi_{b}^{0}\right)$ baryon. The upper limits on the ratio $\mathcal{R}\left(\Lambda_{c}^{+} \pi^{-}\right)$and $\mathcal{R}\left(\Xi_{c}^{+} \pi^{-}\right)$ are set at $95 \%$ confidence level under different mass and lifetime hypothesis for $\Xi_{b c}^{0}$ and $\Omega_{b c}^{0}$ baryons, and shown in Fig. 6.

\section{Summary}

The recent results of beauty-hadron spectroscopy at LHCb are presented in this proceeding, including the observation of new excited $B_{s}^{0}$ states in the $B^{+} K^{-}$final system, the observation of a 

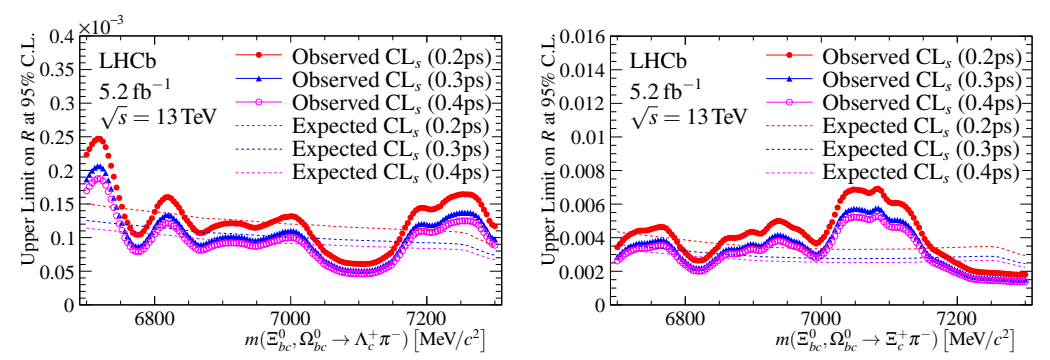

Figure 6: Upper limits on the production ratio for $H_{b c}^{0}$ decays to (left) $\Lambda_{c}^{+} \pi^{-}$and (right) $\Xi_{c}^{+} \pi^{-}$.

new $\Xi_{b}^{0}$ state in $\Xi_{b}^{-} \pi^{+}$final state, the observation of two new excited $\Xi_{b}^{0}$ states decaying to $\Lambda_{b}^{0} K^{-} \pi^{+}$ and a search for doubly heavy baryons $\Xi_{b c}^{0}$ and $\Omega_{b c}^{0}$ decaying to $\Lambda_{c}^{+} \pi^{-}$and $\Xi_{c}^{+} \pi^{-}$. These would improve the understanding of hadronic structure and nonperturbative QCD.

\section{References}

[1] M. Gell-Mann, A schematic model of baryons and mesons, Phys. Lett. 8 (1964) 214

[2] G. Zweig, An $\mathrm{SU}_{3}$ model for strong interaction symmetry and its breaking; Version 1 CERNTH-401, CERN, Geneva, 1964

[3] M. Neubert, Heavy quark symmetry, Phys. Rept. 245 (1994) 259, arXiv: hep-ph/9306320

[4] LHCb collaboration, R. Aaij et al., Observation of new excited $B_{s}^{0}$ states, Eur. Phys. J. C 81 (2021) 601, arXiv:2010.15931

[5] LHCb collaboration, R. Aaij et al., Observation of a new $\Xi_{b}^{-}$resonance, Phys. Rev. Lett. 121 (2018) 072002, arXiv: 1805.09418

[6] LHCb collaboration, R. Aaij et al., Observation of a new $\Xi_{b}^{0}$ state, Phys. Rev. D103 (2021) 012004, arXiv:2010.14485

[7] LHCb collaboration, R. Aaij et al., Observation of new resonances in the $\Lambda_{b}^{0} \pi^{+} \pi^{-}$system, Phys. Rev. Lett. 123 (2019) 152001, arXiv: 1907.13598

[8] B. Chen, S.-Q. Luo, X. Liu, and T. Matsuki, Interpretation of the observed $\Lambda_{b}(6146)^{0}$ and $\Lambda_{b}(6152)^{0}$ states as $1 D$ bottom baryons, Phys. Rev. D100 (2019) 094032, arXiv: 1910.03318

[9] LHCb collaboration, R. Aaij et al., Observation of two new excited $\Xi_{b}^{0}$ states decaying to $\Lambda_{b}^{0} K^{-} \pi^{+}$, arXiv: 2110.04497

[10] LHCb collaboration, R. Aaij et al., Search for the doubly heavy baryons $\Omega_{b c}^{0}$ and $\Xi_{b c}^{0}$ decaying to $\Lambda_{c}^{+} \pi^{-}$and $\Xi_{c}^{+} \pi^{-}$, Chin. Phys. $\mathbf{C 4 5}$ (2021) 093002, arXiv: 2104.04759 